\title{
Artículo Especial: Permanencia y devenir: de la filosofía griega al análisis de decisiones médicas
}

\author{
Permanence and moving: from greek philosophy to medical decision analysis
}

Carlos Musso* ${ }^{*}$ Federico Augustovski ${ }^{\star \star}$ y Alberto Velazquez ${ }^{* \star *}$

\begin{abstract}
Resumen
Haciendo un paralelo con los aportes de Markov a la moderna teoría del análisis de decisión, los autores de este artículo describen las posturas antagónicas de Heráclito, que sostenía que el Universo estaba en un eterno fluir (devenir) y la de Parménides, que sostenía que el movimiento era una ilusión de los sentidos (permanencia) así como la síntesis de Demócrito en su teoría atómica, que combina elementos de ambas como la inmutabilidad de las partículas sub-atómicas y el movimiento de las mismas.
\end{abstract}

\section{Abstract}

The authors of this article describe Heraclito's and Parmenides antagonic viewpoint about the universe, making a parallelism with modern Markov's decision analysis theory. According to Heraclito, the universe was in an eternal flowing state opossite to Parmenides, who believed movement was an ilussion of senses. The authors also mention Democrito's atomic theory, in which elements of both previously mentioned theories and the immutability of subatomic particles and their movement are combined.

Palabras claves: análisis de decisión, teoría atómica, movimiento, inmutabilidad. Key words: decision analysis, atomic theory, movement, immutability.

En el siglo $V$ antes de Cristo, Heráclito de Efeso conmocionó el pensamiento de su época al enunciar que el universo entero estaba sometido a un eterno fluir, de modo que incluso aquello que parecía estático estaba en realidad en perenne mutación. Muchos siglos después, científicos de la talla de Markov reelaboraron el concepto filosófico del devenir, pero desde una perspectiva matemática.

En el campo de la biología estas ideas contribuyeron a replantear la arbitraria diferenciación entre la forma y la función de los órganos, al facilitar la comprensión del hecho de que la anatomía y la fisiología de un parénquima son en realidad distintas velocidades (lenta y rápida respectivamente) de un mismo devenir, en función del cual dicho órgano es minuto a minuto distinto de la versión precedente de sí. Es nuestra falta de sutileza aquello que nos impide aprehender el carácter bifásico de este movimiento.

Por otra parte, los médicos asistenciales presencian a diario el fenómeno del cambio continuo en las enfermedades de sus pacientes, en las propias, y en el recambio de los profesionales intervinientes. Este "movimiento" es la génesis de la incertidumbre en la cual los médicos se encuentran inmersos, en mayor o en menor grado, durante sus actos diagnósticos y terapéuticos. Precisamente en respuesta a dicha incertidumbre es que se desarrolló la disciplina del análisis de decisión, heredera directa de los aportes de Heráclico y Markov.
Sin embargo, otro eminente pensador griego, Parménides, se opuso férreamente al concepto del flujo perenne, al sostener que el movimiento era una ilusión engañosa de los sentidos, pues éstos mostraban aparentes transformaciones de algo que en realidad era una sustancia única, inmóvil e inmutable. Parménides razonaba de la siguiente forma: "si se dice que algo cambia, significa que se convierte en lo que no es, pero como puede algo ser aquello que no es? Significa entonces que en realidad nunca lo fue; luego, no fue la cosa aquello que cambió, sino nuestro conocimiento acerca de su naturaleza".

Asistimos entonces a una fuerte controversia entre las posturas filosóficas de Heráclito (devenir) y de Parménides (permanencia). No obstante, dicha paradoja es aparente como nos lo demuestra la teoría atómica de Demócrito. Este postulado sostiene que el mundo está formado por un número determinado de partículas eternas e inmutables (átomos) las cuales están sujetas a un juego de constante combinación y recombinación, lo cual da cuenta del por qué del nacimiento, la existencia y la disolución de las cosas del mundo.

Desde la óptica de esta teoría permanencia y devenir serían los planos sub y supra atómicos respectivamente del mismo fenómeno. 